\title{
Compostos de biopolietileno/línter de algodão compatibilizados com PE-g-MA
}

\author{
Compounds of biopolyethylene/cotton linter compatibilized with PE-g-MA \\ Compuestos de biopolietileno/linter de algodón compatibles con PE-g-MA
}

\author{
Aline Florindo Salviano \\ ORCID: https://orcid.org/0000-0002-6615-0381 \\ Universidade Federal de Campina Grande, Brasil \\ E-mail: alineflorindo@ymail.com \\ Carlos Bruno Barreto Luna \\ ORCID: https://orcid.org/0000-0002-2441-7439 \\ Universidade Federal de Campina Grande, Brasil \\ E-mail: brunobarretodemaufcg@hotmail.com \\ Danilo Diniz Siqueira \\ ORCID: https://orcid.org/0000-0002-3533-513X \\ Universidade Federal de Campina Grande, Brasil \\ E-mail: danilodinizsiqueira@gmail.com \\ Edcleide Maria Araújo \\ ORCID: https://orcid.org/0000-0003-4906-864X \\ Universidade Federal de Campina Grande, Brasil \\ E-mail: edcleide.araujo@ufcg.edu.br \\ Rodholfo da Silva Barbosa Ferreira \\ ORCID: https://orcid.org/0000-0002-5547-994X \\ Universidade Federal de Campina Grande, Brasil \\ E-mail: rodholfoferreira@gmail.com \\ Elieber Barros Bezerra \\ ORCID: https://orcid.org/0000-0003-1637-7761 \\ Universidade Federal de Campina Grande, Brasil \\ E-mail: elieberbarros@hotmail.com
}

\begin{abstract}
Resumo
Atualmente, os polímeros vêm sendo reforçados com fibras naturais, visando a geração de uma classe de novos materiais menos agressivos ao meio ambiente. Portanto, este trabalho teve como objetivo desenvolver compostos de biopolietileno (BioPE)/línter de algodão, compatibilizados com o polietileno enxertado com anidrido maleico (PE-gMA). Os compostos foram preparados, inicialmente, em uma extrusora de rosca dupla corrotacional e, posteriormente, os grânulos extrudados foram moldados por injeção. As propriedades mecânicas (impacto e tração), temperatura de deflexão térmica (HDT), temperatura de amolecimento Vicat (TAV), calorimetria exploratória diferencial (DSC), ângulo de contato e microscopia eletrônica de varredura (MEV) foram avaliadas. Observou-se um aumento discreto no módulo elástico, na resistência à tração, na HDT e na TAV dos compostos, em relação ao BioPE puro. Todavia, houve perdas na resistência ao impacto. A presença do PE-g-MA e do línter aumentou o grau de cristalinidade dos compostos, em comparação ao BioPE. Os compostos compatibilizados apresentaram partículas de línter conectadas com a matriz de BioPE, indicando possíveis interações entre o anidrido maleico e os grupos hidroxilas do línter. Resultados aprimorados foram alcançados quando se utilizou PE-g-MA com maior grau de enxertia, sugerindo que a funcionalização impacta nas propriedades mecânicas e térmicas dos compostos.
\end{abstract}

Palavras-chave: Biopolietileno; Línter de algodão; Compatibilizante; Compostos; Propriedades.

\begin{abstract}
Currently, polymers have been reinforced with natural fibers, aiming at generating a class of new materials less aggressive to the environment. Therefore, this study aimed to develop biopolyethylene (BioPE)/cotton linter compounds, compatible with polyethylene grafted with maleic anhydride (PE-g-MA). The compounds were initially prepared in a co-rotational twin screw extruder, and thereafter, the extruded granules were injection molded. The compounds were characterized by mechanical properties (impact and traction), heat deflection temperature (HDT), Vicat softening temperature (VST), differential exploration calorimetry (CSD), contact angle and scanning electronic microscope (SEM). There was a slight increase in elastic modulus, tensile strength, HDT and VST of the compounds, compared to neat BioPE. However, there were losses in impact strength. The presence of PE-g-MA and the linter increased the degree of crystallinity of the compounds, compared to BioPE. The compatibilized compounds showed particles of linter connected with the BioPE matrix, indicating possible interactions between maleic anhydride and hydroxyl groups of the linter. Improved results were achieved when PE-g-MA was used with a higher degree of grafting, suggesting that functionalization impacts the mechanical and thermal properties of the compounds.
\end{abstract}

Keywords: Biopolyethylene; Cotton linter; Compatibilizer; Compounds; Properties. 


\begin{abstract}
Resumen
Actualmente, los polímeros se están reforzando con fibras naturales, con el objetivo de generar una clase de nuevos materiales que sean menos dañinos para el medio ambiente. Por lo tanto, este trabajo tuvo como objetivo desarrollar compuestos de fibra de algodón/ biopolietileno (BioPE), compatibles con polietileno injertado con anhídrido maleico (PE-g-MA). Los compuestos se prepararon inicialmente en una extrusora de doble husillo co-rotacional y posteriormente se moldearon por inyección los gránulos extruidos. Se evaluaron las propiedades mecánicas (impacto y tracción), temperatura de deflexión térmica (HDT), temperatura de ablandamiento Vicat (TAV), calorimetría diferencial de barrido (DSC), ángulo de contacto y microscopía electrónica de barrido (SEM). Se observó un aumento discreto en el módulo elástico, la resistencia a la tracción, HDT y TAV de los compuestos, en comparación con BioPE puro. Sin embargo, hubo pérdidas en la resistencia al impacto. La presencia de PE-g-MA y linter aumentó el grado de cristalinidad de los compuestos en comparación con BioPE. Los compuestos compatibilizados presentaron partículas intermedias conectadas con la matriz de BioPE, lo que indica posibles interacciones entre el anhídrido maleico y los grupos hidroxilo intermedios. Se lograron mejores resultados cuando se utilizó PE-g-MA con un mayor grado de injerto, lo que sugiere que la funcionalización impacta en las propiedades mecánicas y térmicas de los compuestos.

Palabras clave: Biopolietileno; Linter de algodón; Compatibilizador; Compuestos; Propiedades.
\end{abstract}

\title{
1. Introdução
}

Atualmente, a sociedade está preocupada em preservar o meio ambiente e, por isso, cientistas e pesquisadores concentraram suas atenções na busca de soluções para as grandes quantidades de resíduos que são produzidas diariamente, principalmente devido ao uso de produtos derivados de materiais poliméricos (Taghavi et al., 2018; Luna et al., 2019a; Beydoun \& Klankermayer, 2020; Santos Filho et al., 2021). Nesse sentido, métodos sustentáveis foram desenvolvidos para estimular "tecnologias verdes", ou seja, o desenvolvimento de materiais que favoreça um ciclo ambiental fechado, produzindo resíduos que minimizem os efeitos nos ecossistemas (Siqueira et al., 2019a; Siqueira et al., 2020; Cataldi et al., 2020).

O uso de biopolímeros produzidos a partir de matérias-primas renováveis, como milho, cana-de-açúcar, celulose, quitina e outros, é considerado uma possível solução para reduzir a poluição ambiental (Ferreira et al., 2021). O biopolietileno (Bio-PE) produzido a partir de etanol derivado da cana-de-açúcar, embora não seja biodegradável, mantém o equilíbrio de dióxido de carbono $\left(\mathrm{CO}_{2}\right)$ na natureza (Bezerra et al., 2019a). Além de ser um material ecológico, o Bio-PE apresenta as mesmas propriedades técnicas e processáveis da resina feita a partir de fontes fósseis (Brito et al., 2012; Luna et al., 2021). Por isso, o desenvolvimento de compósitos com matriz polimérica de Bio-PE é considerado primordial, tanto pelo potencial de aplicação tecnológica, como pela diminuição dos impactos ambientais (Castro et al., 2012; Silva et al., 2021).

O desenvolvimento de compósitos de Bio-PE reforçados com fibras naturais apresenta potencial, uma vez que eles apresentam boas propriedades mecânicas, baixo custo e baixa densidade (Castro et al., 2013). Entretanto, as fibras naturais de origem vegetal são compostas basicamente de celulose, lignina e hemicelulose, de maneira que a celulose é o componente principal, a qual apresenta radicais hidroxilas (-OH) (Mei \& Oliveira, 2017). Por tal razão, não há boa afinidade entre as fibras naturais e as matrizes poliméricas e, por consequência, as propriedades mecânicas são fracas (Dong, 2017). Para melhorar a adesão entre a fibra natural e a matriz polimérica, uma alternativa seria a adição de um agente compatibilizante (Montanes et al., 2018). Esses compatibilizantes apresentam partes polares e partes apolares na sua estrutura química, e são utilizados para promover a formação de ligações químicas entre a superfície da fibra natural e a matriz polimérica (Poletto, 2017a). O agente compatibilizante mais utilizado em compósitos poliméricos com fibras naturais consiste em polímero modificado com anidrido maleico (Poletto, 2018; Morais et al., 2019).

Existem várias fibras naturais sendo exploradas como cargas em matrizes poliméricas, como juta, sisal, algodão, linho, fibra de palmeira, fibra de coco, fibras feitas de celulose regenerada e curauá (Mei \& Oliveira, 2017). Todavia, não se localizaram trabalhos baseados no biopolietielno (bio-PE) com fibras derivadas do algodão.

Portanto, este trabalho teve como objetivo desenvolver compostos de biopolietileno reforçados com línter de algodão, utilizando-se o polietileno enxertado com anidrido maleico (PE-g-MA) como agente compatibilizante, com alto e baixo teor de anidrido maleico (MA). 


\section{Revisão Bibliográfica}

Os produtos de polímeros à base de petróleo ainda são dominantes no mercado mundial, devido as suas boas propriedades mecânicas e térmicas, bem como a sua grande versatilidade em diversas aplicações (Luna et al., 2019b). Contudo, dadas as características de não biodegradabilidade e durabilidade das poliolefinas, um sério problema ambiental segue a sociedade contemporânea, com acúmulo de lixos (Bezerra et al., 2019b). Portanto, a sociedade vem solicitando ao setor industrial a adoção de medidas "ecologicamente aceitáveis", com o uso racional dos recursos naturais, principalmente na produção de materiais para os setores produtivos (Hoffman et al., 2019; Siqueira et al., 2019b).

Atualmente, há um grande crescimento na procura por materiais compósitos que utilizam fibras naturais, visto que são menos poluentes, em comparação com as fibras sintéticas (Marinho et al., 2018; Ferreira et al., 2019; Arabeche et al., 2020). O interesse no uso de fibras naturais como reforço de materiais compósitos está em constante ascensão, principalmente por causa das múltiplas vantagens associadas: são naturais, renováveis e atóxicas, são de fácil disponibilidade, apresentam baixa densidade, são biodegradáveis, apresentam custo baixo, alta rigidez e, ao mesmo tempo, são menos abrasivas (Jesus et al., 2019; Kotik, 2019; Padilha e Zard, 2010). Essas propriedades chamaram atenção da comunidade científica e da indústria, visando o desenvolvimento de novos materiais que contribuam para um ciclo sustentável e, gerando, os chamados “ecocompósitos" (Cicconi, 2020; Wis et al., 2020).

Bezerra et al. (2015) desenvolveram compósitos de policaprolactona (PCL)/nanolínter, com os teores de 3 e $5 \%$ em peso da carga natural. O ensaio de tração demonstrou que a presença da nanolínter promoveu um pequeno aumento no módulo elástico. Contudo, houve uma redução na resistência ao impacto com a presença do nanolínter. A temperatura de deflexão térmica (HDT) apresentou um aumento significativo para os compósitos, em média $10^{\circ} \mathrm{C}$, em comparação com a PCL pura.

Castro et al. (2017) investigaram o potencial da fibra de carauá como carga de reforço na matriz polimérica de biopolietileno (BioPE), utilizando-se os óleos de mamona (OM) e de canola (CA) como compatibilizantes das fibras polares e da matriz apolar. Os resultados indicaram que a incorporação de óleos em várias composições, particularmente o óleo de mamona, geralmente conduziu à melhores propriedades mecânicas, em comparação ao compósito BioPE/fibra carauá sem óleo. Em geral, a utilização de óleo apresenta potencial como um compatibilizante na interface matriz/fibra.

Bosenbecker et al. (2018) avaliaram as propriedades térmicas de compósitos de polietileno de alta densidade reforçados com a celulose obtida a partir da casca de arroz. Os compósitos obtidos foram preparados nas proporções de 5, 10 e $15 \%$ em massa de celulose, utilizando-se para isso uma extrusora de rosca simples. O método aplicado para a obtenção da celulose a partir da casca de arroz foi eficiente, revelando um aumento da cristalinidade após tratamentos químicos. Os compósitos apresentaram um ligeiro aumento na estabilidade térmica em relação à matriz de polietileno, no entanto, não foi observada uma mudança significativa nas temperaturas de fusão e de cristalização para as amostras estudadas. A casca de arroz pode ser reaproveitada para o desenvolvimento de compósitos com boas propriedades térmicas, contribuindo para o reaproveitamento de um resíduo.

A influência da granulometria do pó do talo de bananeira foi investigada, visando o desenvolvimento de compósitos de polipropileno (PP)/talo de bananeira (TB) na proporção 90/10\% em peso, por Seixas et al. (2018). Os resultados dos ensaios de tração mostraram que os compósitos apresentaram baixo desempenho mecânico, o que pode ser atribuído a pouca interação entre fibra/matriz. Apesar da variação granulométrica das fibras não influenciar nas propriedades mecânicas dos compósitos, foi possível observar que a adição das fibras melhorou a estabilidade térmica do material, especialmente nas granulometrias mais finas e a temperatura do ponto máximo de perda de massa chegou a aumentar, em relação ao PP puro. Os compósitos de PP/talo de bananeira apresentam boas propriedades térmicas e com propriedades promissoras para aplicações que não requerem elevada resistência mecânica.

Compósitos verdes de polipropileno (PP)/sabugo de milho (SM) foram desenvolvidos por Ramos et al. (2019). Os 
compósitos com até $20 \%$ em peso de sabugo de milho apresentaram partículas uniformemente dispersas na matriz de PP. A resistência à tração e o alongamento na ruptura dos compósitos diminuíram, enquanto o módulo de elasticidade sob tração e flexão aumentaram, em comparação ao PP puro. A resistência ao impacto permaneceu praticamente constante, ou seja, em nível equiparável ao PP. A termogravimetria (TG) mostrou que o sabugo de milho é termicamente estável até $200{ }^{\circ} \mathrm{C}$, garantindo que nenhuma degradação ocorreu durante o processamento. Os compósitos de PP/SM são mais cristalinos em relação ao PP, indicando que o sabugo de milho atua como agente nucleante.

O desenvolvimento e a comercialização de compósitos produzidos a partir de fontes naturais são importantes, visto que permitirão a redução da dependência de materiais fabricados com recursos não renováveis (Gandara et al., 2017; Luna et al., 2019c; Santos et al., 2020). Ao mesmo tempo, o desenvolvimento de materiais compósitos poderá reduzir custos, com bom desempenho mecânico e redução do impacto ambiental (Silva et al., 2018; Nery et al., 2018). Nesse contexto, torna-se importante avaliar o potencial das cargas naturais derivadas do algodão, visto que poucos trabalhos foram reportados na literatura científica de polímeros e compósitos.

\section{Metodologia}

\section{Materiais}

O polietileno de alta densidade verde (BioPE), comercializado com o código SHC7260® e índice de fluidez (IF) de 7,2 g/10 min $\left(190^{\circ} \mathrm{C} / 2,16 \mathrm{~kg}\right)$, produzido a partir da cana-de-açúcar e fornecido na forma de pellets, pela Braskem. O línter de algodão (LA) foi utilizado como carga, na forma de pó, produzido e cedido pela Embrapa Agroindústria Tropical, sediada na cidade de Fortaleza-CE. O polietileno enxertado com anidrido maleico (PE-g-MA) foi utilizado como agente compatibilizante, comercializado com o código 3009®, contendo baixo grau de enxertia de anidrido maleico, na forma de pellets e fornecido pela Polybond. O polietileno enxertado com anidrido maleico (PE-g-MA) foi utilizado como agente compatibilizante, comercializado com o código $3029 \circledR$, contendo alto grau de enxertia de anidrido maleico, na forma de pellets e fornecido pela Polybond

\section{Métodos}

\section{Preparação do Concentrado}

Os concentrados poliméricos do BioPE/línter de algodão foram preparados pelo método de intercalação por fusão, usando um misturador de alta rotação (Homogeneizador Termocinético), modelo MH-50H. O equipamento foi acionado durante um período de aproximadamente 15 segundos, ocorrendo à mistura e a fusão dos concentrados por atrito. Posteriormente, o concentrado BioPE/línter de algodão foi triturado em moinho de facas, dando origem aos flakes.

\section{Processamento por Extrusão}

Os compostos foram misturados a seco e, subsequentemente, processados em uma extrusora de rosca dupla corrotacional modular, modelo ZSK $(\mathrm{D}=18 \mathrm{~mm}$ e L/D $=40)$, da Coperion Werner-Pfleiderer. O perfil de temperatura utilizado na extrusora foi de $180^{\circ} \mathrm{C}-180^{\circ} \mathrm{C}-180^{\circ} \mathrm{C}-180^{\circ} \mathrm{C}-180^{\circ} \mathrm{C}-180^{\circ} \mathrm{C}-180^{\circ} \mathrm{C}$, velocidade de rotação da rosca de 250 rpm e taxa de alimentação controlada de $4 \mathrm{~kg} / \mathrm{h}$, com perfil de rosca configurado com elementos de misturas distributivos e dispersivos. O material granulado foi seco em uma estufa com vácuo por $24 \mathrm{~h}$, em temperatura de $60^{\circ} \mathrm{C}$. O BioPE foi processado e seco nas mesmas condições dos compostos. A Tabela 1 apresenta as composições que foram formuladas dos compostos poliméricos, com as proporções em massa (\%). 
Tabela 1. Composições dos compostos com as proporções em massa (\%). * Alto grau de enxertia; ** Baixo grau de enxertia.

\begin{tabular}{l|c|c|c}
\hline \multicolumn{1}{c|}{ Amostras } & BioPE (\% em massa) & LA (\% em massa) & PE-g-MA (\% em massa) \\
\hline BioPE & 100 & - & - \\
BioPE/LA & 95 & 5 & - \\
BioPE/LA/PE-g-MA (A)* & 85 & 5 & 10 \\
BioPE/LA/PE-g-MA (B)** & 85 & 5 & 10 \\
\hline
\end{tabular}

Fonte: Dados da pesquisa.

\section{Moldagem por Injeção}

O BioPE e os compostos foram moldados por injeção, em uma injetora Arburg, Modelo Allrounder 207C Golden Edition, para a obtenção de corpos de prova de impacto, tração e HDT, segundo as normas ASTM D256, ASTM D638 e ASTM D648, respectivamente. As condições de moldagem foram: perfil de temperatura de $180 ; 180 ; 180 ; 180 ; 180^{\circ} \mathrm{C}$; temperatura do molde de $20^{\circ} \mathrm{C}$; tempo de resfriamento no molde de 25 s; pressão de injeção e recalque, 1000 e 500 bar, respectivamente.

\section{Caracterização dos Materiais}

O ensaio de resistência ao impacto Izod foi realizado em corpos de prova entalhados, segundo a norma ASTM D256, em um aparelho da marca Ceast modelo Resil 5,5 J, operando com martelo de 2,75 $\mathrm{J}$, em temperatura ambiente $\left(\sim 23^{\circ} \mathrm{C}\right) . \mathrm{Os}$ resultados foram analisados com uma média de sete corpos de prova.

O ensaio de tração foi realizado em corpos de prova injetados, segundo a norma ASTM D638, utilizando uma máquina de ensaios universal da marca EMIC DL 2000, com velocidade de $50 \mathrm{~mm} / \mathrm{min}$ e célula de carga de $20 \mathrm{kN}$, em temperatura ambiente $\left(\sim 23^{\circ} \mathrm{C}\right)$. Os resultados foram analisados com uma média de sete corpos de prova.

A temperatura de deflexão térmica (HDT) foi obtida conforme a norma ASTM D648, em um equipamento Ceast, modelo HDT 6 VICAT, com uma tensão de $455 \mathrm{kPa}$ e taxa de aquecimento de $120^{\circ} \mathrm{C} / \mathrm{h}$. A temperatura foi determinada após a amostra ser defletida 0,25 mm. Os resultados foram analisados com uma média de três corpos de prova.

O ensaio de temperatura de amolecimento Vicat foi realizado segundo a norma ASTM D1525, em um equipamento Ceast, modelo HDT 6 VICAT, empregando-se uma taxa de aquecimento de $120^{\circ} \mathrm{C} / \mathrm{h}$ e uma carga de $50 \mathrm{~N}$. A temperatura foi determinada após a agulha penetrar $1 \mathrm{~mm}$ nos corpos de prova. Os resultados foram analisados com uma média de três corpos de prova.

A análise de calorimetria exploratória diferencial (DSC) foi realizada em um aparelho DSC-Q20 da TA Instruments. $\mathrm{O}$ ensaio foi realizado partindo-se de 30 até $100^{\circ} \mathrm{C}$, sob taxa de aquecimento de $10^{\circ} \mathrm{C} / \mathrm{min}$, vazão do gás de $50 \mathrm{~mL} / \mathrm{min}$, atmosfera de nitrogênio e empregando $6 \mathrm{mg}$ de amostra.

A análise do ângulo de contato foi realizada pelo método da gota séssil, por meio de um ângulo de contato portátil, modelo Phoenix-i da Suface Eletro Optics - SEO. A gota foi depositada sobre amostra de teste de impacto fazendo uso de um dosador micrométrico, sendo a imagem captada e analisada pelo software associado ao equipamento.

As análises por microscopia eletrônica de varredura (MEV) foram realizadas na superfície de fratura dos corpos de prova submetidos ao ensaio de impacto. Utilizou-se um microscópio eletrônico de varredura, Shimadzu SSX-550 Superscan, a uma voltagem de $30 \mathrm{kV}$, sob alto vácuo. As superfícies de fratura das amostras foram revestidas com ouro.

\section{Resultados e Discussão}

Microscopia Eletrônica de Varredura (MEV)

A Figura 1 (a,b,c,d) ilustra as micrografias obtidas por MEV do BioPE, do BioPE/LA e dos compostos compatibilizados BioPE/LA/PE-g-MA, contendo baixo e alto grau de enxertia, respectivamente. Observa-se, na Figura 1 (a), 
que a superfície do BioPE apresentou um aspecto regular de fratura dúctil, ou seja, com uma ampla deformação plástica, confirmando a boa resistência ao impacto que será apresentada posteriormente. Na Figura 1(b), o composto BioPE/LA apresentou muitos vazios na matriz de BioPE, o que indica uma fraca adesão interfacial entre o línter de algodão (LA) e o BioPE. A má adesão ocorre devido à alta tensão interfacial existente entre os componentes, provavelmente, devido à natureza hidrofílica da fibra e hidrofóbica da matriz de BioPE, o que torna a interface com baixa resistência, corroborando o menor desempenho sob impacto (Liu et al., 2013; Spadetti et al., 2017).

A Figura $1(\mathrm{c}, \mathrm{d})$ apresenta uma redução significativa no nível de vazios, sugerindo que o compatibilizante PE-g-MA aumentou a adesão interfacial entre o BioPE e o línter de algodão (LA). O composto BioPE/LA/PE-g-MA com maior grau de enxertia promoveu uma maior estabilidade na morfologia, visto que o número de vazios é menos pronunciado, em comparação ao de menor grau de enxertia. Isso indica que o maior teor de anidrido maleico no PE-g-MA aumentou a interação com a fibra de línter, uma vez que há maior grupo funcional para reagir com as hidroxilas (Poletto, 2017b). Nesse caso, a tensão aplicada é mais bem distribuída da matriz para a fibra, melhorando o desempenho sob impacto e módulo elástico, como verificado mais adiante. Aparentemente, os compostos BioPE/LA/PE-g-MA apresentam partículas de línter conectadas à matriz de BioPE (círculo preto), reforçando a hipótese de possível reação entre o anidrido maleico e as hidroxilas do línter e, ao mesmo tempo, confirmando o maior desempenho sob impacto desses sistemas, em relação ao composto BioPE/LA. A Figura 2 apresenta a reação proposta para os compostos BioPE/LA/PE-g-MA.

Figura 1. Fotomicrografias obtidas por MEV da superfície de fratura: (a) BioPE (ampliação de 1000x); (b) BioPE/LA; (c) BioPE/LA/PE-g-MA (baixo); (d) BioPE/LA/PE-g-MA (alto). Os compostos apresentam ampliação de 8000x.
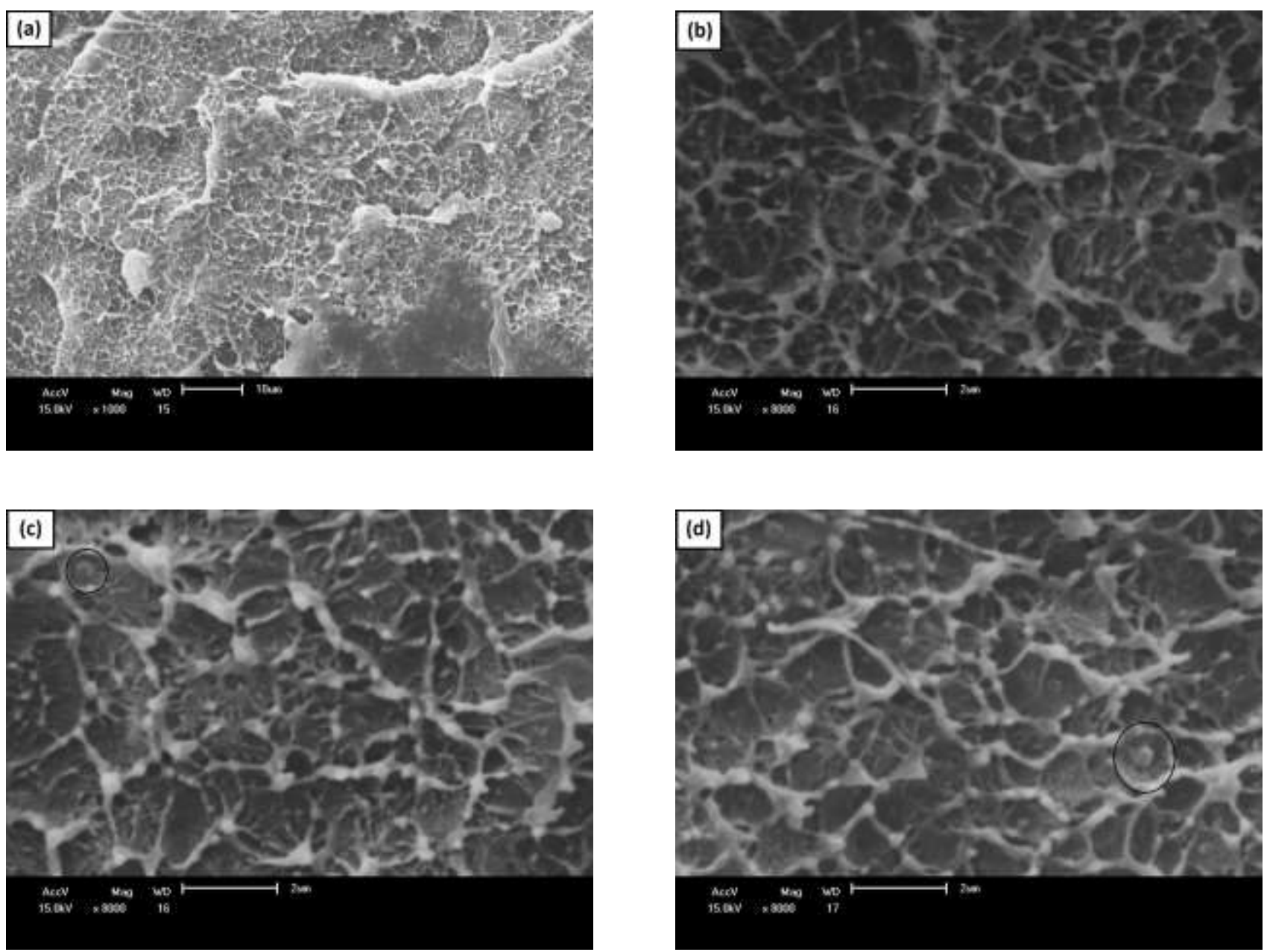

Fonte: Dados da pesquisa. 
Figura 2. Reação proposta para o composto BioPE/LA/PE-g-MA.

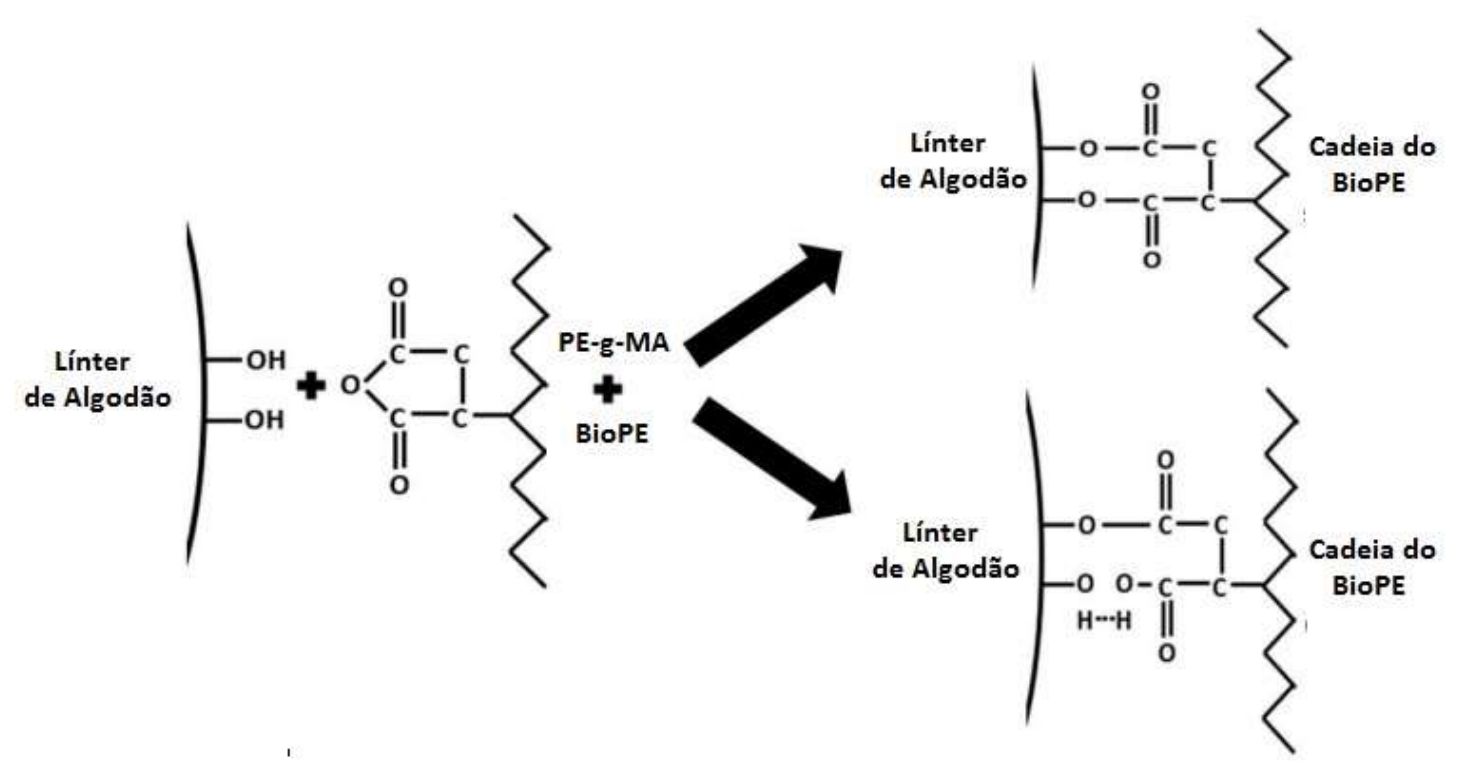

Fonte: Adaptado de Baptista e Canevarolo (2019).

\section{Ângulo de contato}

A Figura 3 apresenta os resultados da análise de ângulo de contato da superfície do BioPE e dos compostos. O BioPE possui ângulo de contato de $62,9^{\circ}$, indicando ser o material mais hidrofóbico. Os compostos, independentemente de serem compatibilizados ou não, apresentaram uma redução do ângulo de contato, em comparação ao BioPE puro. Isso indica que os compostos apresentam uma maior interação com a água, ou seja, são mais hidrofílicos. Quando incorporado o agente compatibilizante (PE-g-MA), os valores do ângulo de contato nos compostos BioPE/LA/PE-g-MA diminuem em comparação ao composto BioPE/LA, indicando uma maior molhabilidade e, consequentemente, melhor adesão, como verificado no MEV. Nesse caso, os compostos compatibilizados apresentam uma tendência de aumentar a interação da fibra com a matriz de BioPE, implicando em um aumento no desempenho mecânico (Catto et al., 2014). 
Figura 3. Ângulo de contato na superfície dos materiais no decorrer de 200 segundos, para: (a) BioPE; (b) BioPE/LA; (c) BioPE/LA/PE-g-MA (baixo); (d) BioPE/LA/PE-g-MA (alto).

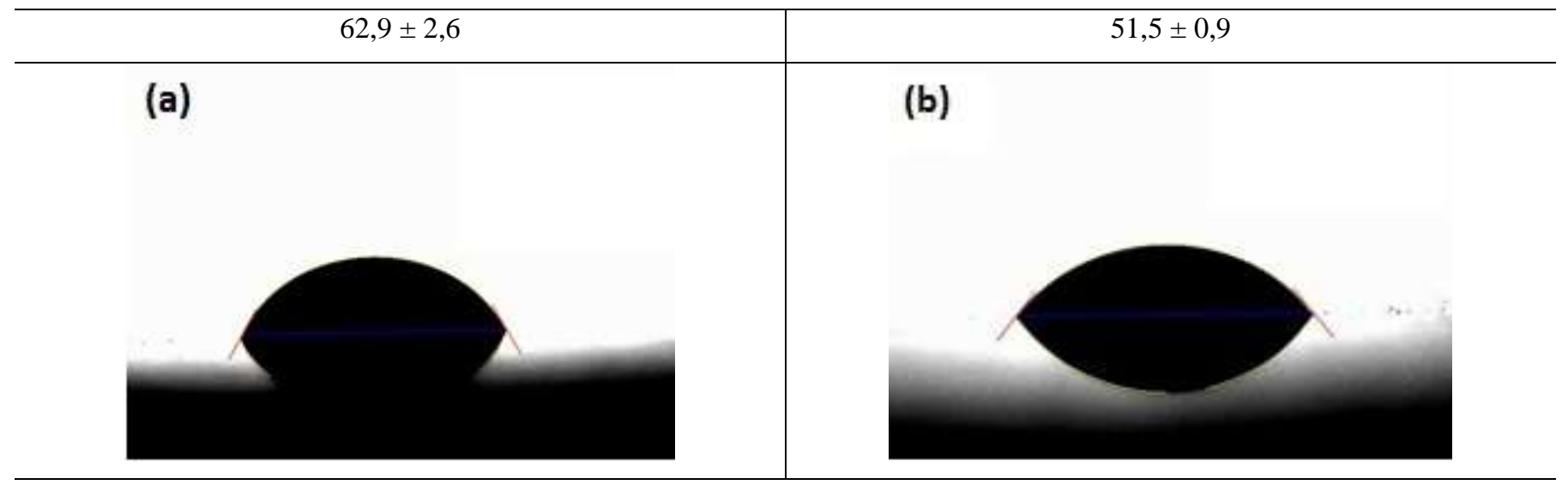

(c)

\section{Calorimetria Exploratória Diferencial (DSC)}

Os parâmetros de fusão, cristalização e o grau de cristalinidade do BioPE e dos compostos estão sumarizados na Tabela 2. As curvas de DSC obtidas durante o segundo ciclo de aquecimento podem ser visualizadas na Figura 4 (a,b). Na Tabela 2, é possível visualizar um único pico de fusão em torno de $134^{\circ} \mathrm{C}$, comumente encontrado em matriz polimérica de polietileno (Firmino et al., 2017). Pode-se observar que a temperatura de fusão (Tm) dos compostos não sofreu alteração significativa em relação ao BioPE, somente sutis alterações. Devido ao baixo teor de línter (LA), a entalpia de fusão ( $\Delta \mathrm{Hf}$ ) praticamente não foi modificada em comparação ao BioPE. Consequentemente, impactou em uma manutenção (BioPE/LA) ou na elevação da cristalinidade global dos compostos compatibilizados, em relação ao BioPE.

Tabela 2. Parâmetros de fusão e de cristalização do BioPE e dos compostos. $* \mathrm{~T}_{\mathrm{m}}=$ Temperatura do pico de fusão; $\mathrm{T}_{\mathrm{C}}=$ Temperatura de cristalização; $\Delta \mathrm{H}_{\mathrm{f}}=$ Entalpia de fusão; $\mathrm{Xc}=$ Grau de cristalinidade, $\mathrm{X}_{\mathrm{C}}=\Delta \mathrm{H}_{\mathrm{f}} / \mathrm{w}^{*} \Delta \mathrm{H}_{\mathrm{o}}$, onde: $\mathrm{w}=$ fração mássica do BioPE; $\Delta \mathrm{H}_{0}=$ Entalpia de fusão do BioPE com 100\% de cristalinidade, $293 \mathrm{~J} / \mathrm{g}$ (Castro et al., 2013).

\begin{tabular}{c|c|c|c|c}
\hline Amostras $^{*}$ & $\mathbf{T}_{\mathbf{m}}\left({ }^{\circ} \mathbf{C}\right)$ & $\mathbf{T}_{\mathbf{c}}\left({ }^{\circ} \mathbf{C}\right)$ & $\boldsymbol{\Delta H}_{\mathbf{f}}(\mathbf{J} / \mathbf{g})$ & $\mathbf{X}_{\mathbf{c}} \mathbf{( \% )}$ \\
\hline BioPE & 134,7 & 114,8 & 183,3 & 62,6 \\
BioPE/LA & 134,9 & 116,4 & 171,8 & 61,7 \\
BioPE/LA/PE-g-MA (baixo) & 133,9 & 117,6 & 181,2 & 65,1 \\
BioPE/LA/PE-g-MA (alto) & 133,1 & 117,7 & 181,9 & 65,3 \\
\hline
\end{tabular}


Figura 4. Curvas DSC para o BioPE puro e os compostos: (a) temperatura de fusão e (b) temperatura de cristalização.
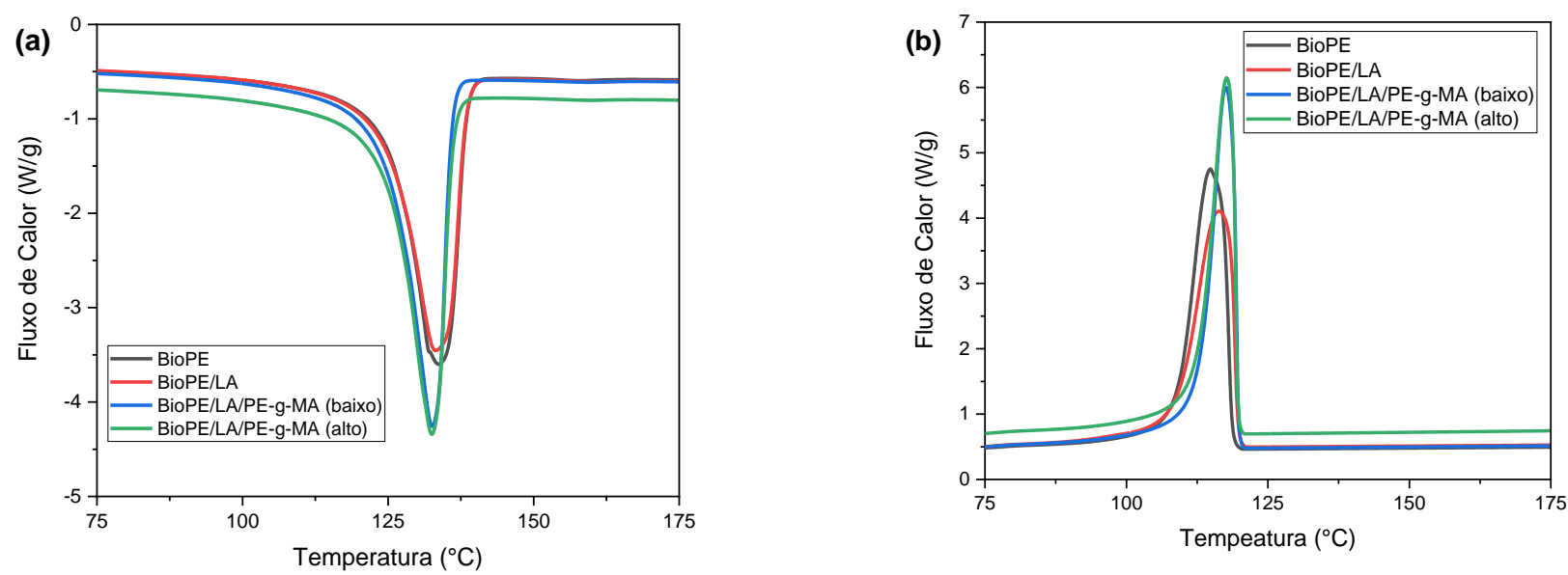

Fonte: Dados da pesquisa.

A temperatura de cristalização dos compostos BioPE/LA e BioPE/LA/PE-g-MA aumentou em relação ao BioPE puro, indicando que o línter acelera o processo de cristalização do BioPE. Ao mesmo tempo, na Figura 4(b), é perceptível o aumento da intensidade dos picos de cristalização dos compostos BioPE/LA/PE-g-MA, quando comparado ao BioPE puro e o composto BioPE/LA. Portanto, os compostos compatibilizados requerem mais energia para promover a cristalização, corroborando o aumento da cristalinidade (Zhang et al., 2019).

Na Tabela 2, observa-se que o grau de cristalinidade do BioPE foi da ordem de 62,6\%, enquanto que a adição de línter (BioPE/LA) não alterou de forma significativa a cristalinidade, ocorrendo somente uma manutenção. Os compostos compatibilizados (BioPE/LA/PE-g-MA), independentemente do grau de enxertia, aumentaram a cristalinidade em comparação ao BioPE e o composto BioPE/LA. Aparentemente, o aumento da interação das fases, com a adição do PE-g-MA no sistema BioPE/LA, favoreceu para que o línter de algodão atuasse como um agente nucleante, contribuindo para o aumento no grau de cristalinidade. Essa suposição é corroborada com a morfologia obtida por MEV, com o aparecimento de conexões interligando a matriz com a carga (Figura 1d). De fato, um dos requisitos essenciais para um bom agente de nucleação é a maior interação entre a matriz polimérica e a carga (Rabello, 2000).

\section{Resistência ao Impacto Izod}

A Figura 5 mostra a resistência ao impacto do BioPE, do BioPE/LA e dos compostos BioPE/LA/PE-g-MA, contendo baixo e alto grau de enxertia, respectivamente. O BioPE apresentou resistência ao impacto na ordem de $100 \mathrm{~J} / \mathrm{m}$, valor típico de polímero dúctil, corroborando a morfologia obtida por MEV. O composto BioPE/LA reduziu a resistência ao impacto em torno de 52,6\%, em comparação ao BioPE puro. Isso ocorreu devido à adição do línter de algodão (LA), uma vez que é uma carga rígida e, consequentemente, tendeu a fragilizar a matriz dúctil. As cargas atuam como concentradores de tensão, restringindo a mobilidade da matriz, reduzindo a deformação plástica (Ramos et al., 1993). 
Figura 5. Resistência ao impacto Izod do BioPE e dos compostos.

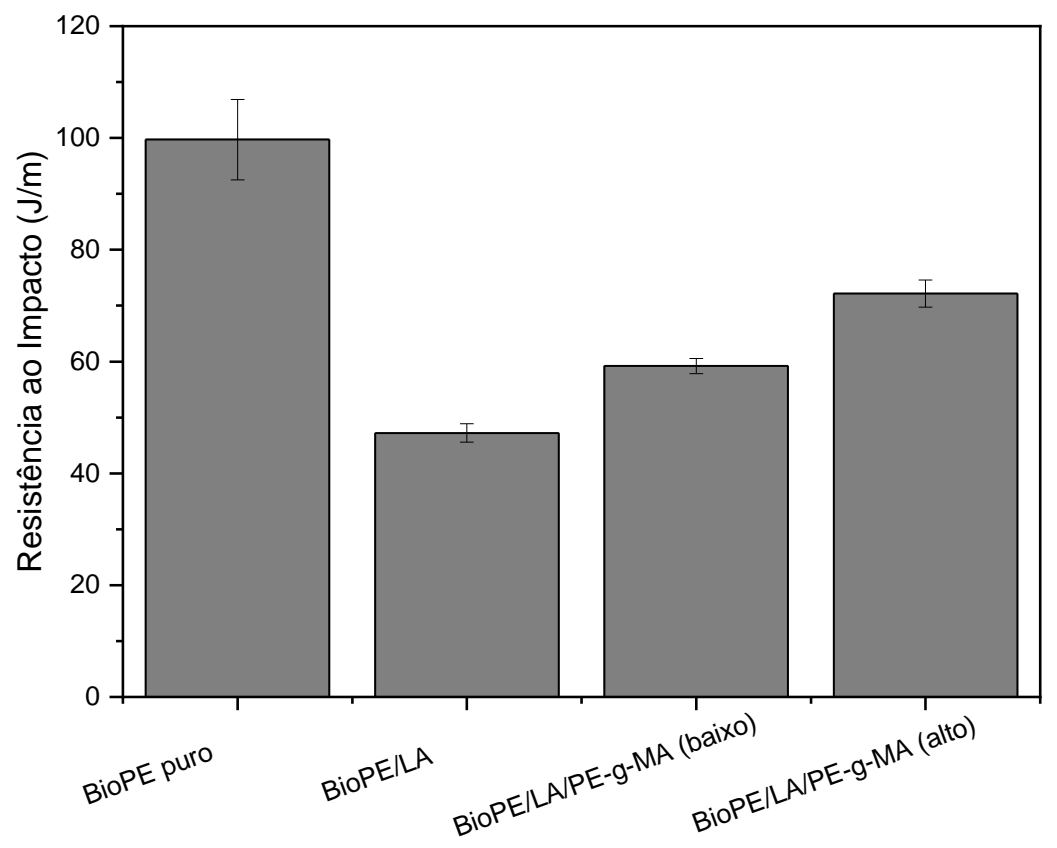

Fonte: Dados da pesquisa.

A adição de $10 \%$ do compatibilizante PE-g-MA promoveu um aumento na resistência ao impacto, em relação ao composto BioPE/LA. O acréscimo nessa propriedade é tomado como um indicativo de que a adição de PE-g-MA gerou uma interação entre as fases presentes, fortalecendo a interface do sistema, o que é fundamental para a transferência de tensões entre as fases (Redighieri e Costa, 2008). Ao que tudo indica, possivelmente está havendo formação de ligações covalentes entre os grupos anidrido maleico e os grupos hidroxila presentes na celulose, gerando um aumento no desempenho mecânico dos compósitos, como sugerido por Santos et al. (2010). Aparentemente, o teor de anidrido maleico influenciou a resistência ao impacto, uma vez que o composto BioPE/LA/PE-g-MA com alto grau de enxertia apresentou o maior desempenho sob impacto, em comparação ao de baixo de grau de enxertia. É razoável sugerir que a maior quantidade de anidrido maleico ampliou as interações com as hidroxilas do línter, como verificado na morfologia da Figura 1 (d). Como consequência, aumentou a eficiência da dissipação de energia sob impacto.

\section{Propriedades de tração}

A Figura 6 apresenta os resultados de módulo elástico do BioPE, do BioPE/LA e dos compostos BioPE/LA/PE-gMA, contendo baixo e alto grau de enxertia, respectivamente. 
Figura 6. Módulo elástico do BioPE e dos compostos.

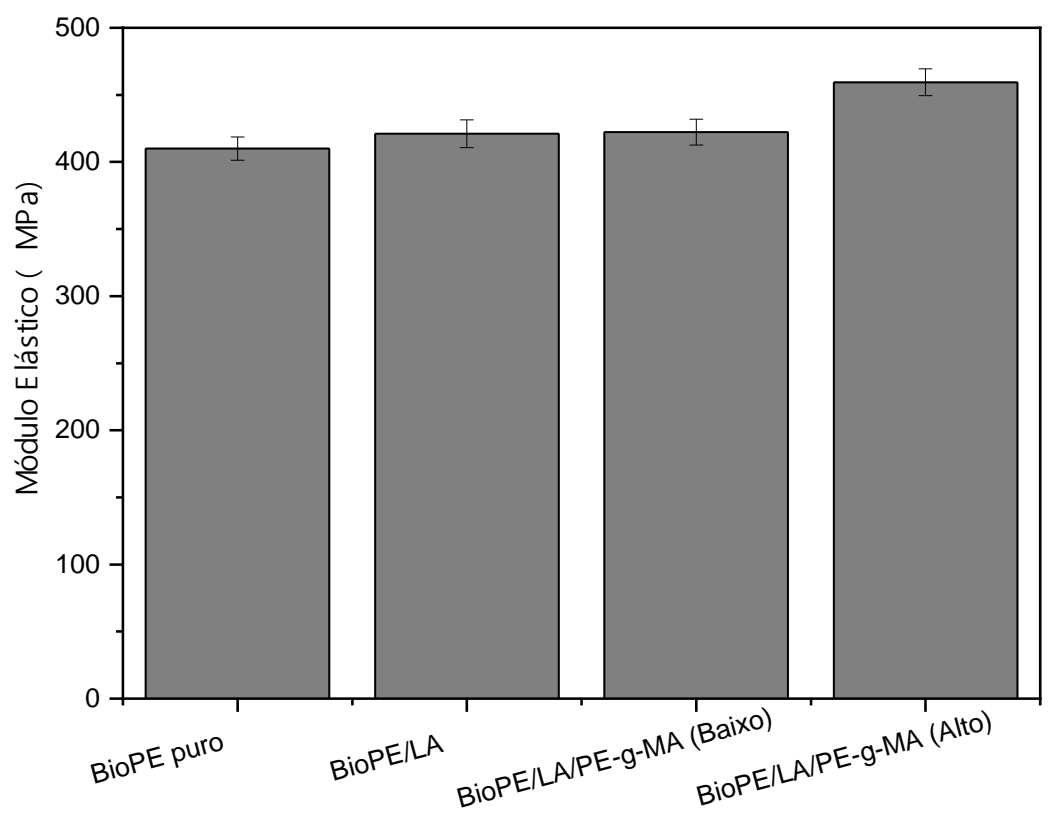

Fonte: Dados da pesquisa.

O BioPE apresentou o menor módulo elástico ( 410 MPa), enquanto o maior desempenho foi alcançado para o composto PE/LA/PE-g-MA com alto teor de enxertia (459,4 MPa). Embora não seja um acréscimo alto, os compostos tenderam a aumentar o módulo elástico, em comparação ao BioPE puro. Isso ocorreu devido ao fato do línter de algodão (LA) ser mais rígido do que a matriz de BioPE e impedir a mobilidade livre do mesmo. Novamente, o composto PE/LA/PE-g-MA com alto teor de enxertia apresenta indícios de interações mais eficientes, reforçando a eventual reação da Figura 2.

Os resultados de resistência à tração do BioPE e dos compostos estão mostrados na Figura 7. Observa-se que a resistência à tração dos compostos, independentemente de serem compatibilizados ou não, não sofreu variação significativa em relação ao BioPE puro. O composto compatibilizado com alto grau de enxertia apresentou o aumento mais evidente nessa propriedade. Contudo, deve-se levar em conta que a adição de línter no BioPE não está reduzindo a tensão máxima, como verificado em outros biocompósitos (Yang et al., 2006). 
Figura 7. Resistência à tração do BioPE e dos compostos.

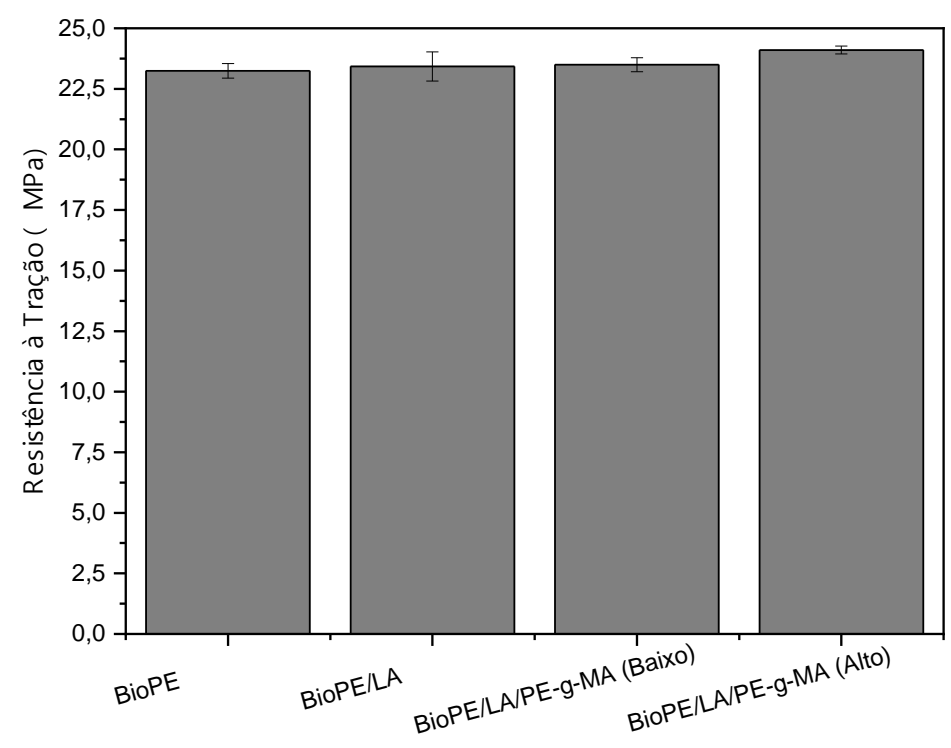

Fonte: Dados da pesquisa.

\section{Temperatura de Deflexão Térmica (HDT)}

A temperatura de deflexão térmica (HDT) é uma propriedade importante na área de polímeros, uma vez que indica a estabilidade dimensional dos materiais quando submetidos simultaneamente à temperatura e uma carga fixa (Luna et al., 2019d; Daghigh et al., 2020; Kim e Cho, 2020). A Figura 8 mostra os resultados da temperatura de deflexão térmica do BioPE, do BioPE/LA e dos compostos BioPE/LA/PE-g-MA, em função do grau de enxertia.

Figura 8. Temperatura de deflexão térmica (HDT) do BioPE e dos compostos.

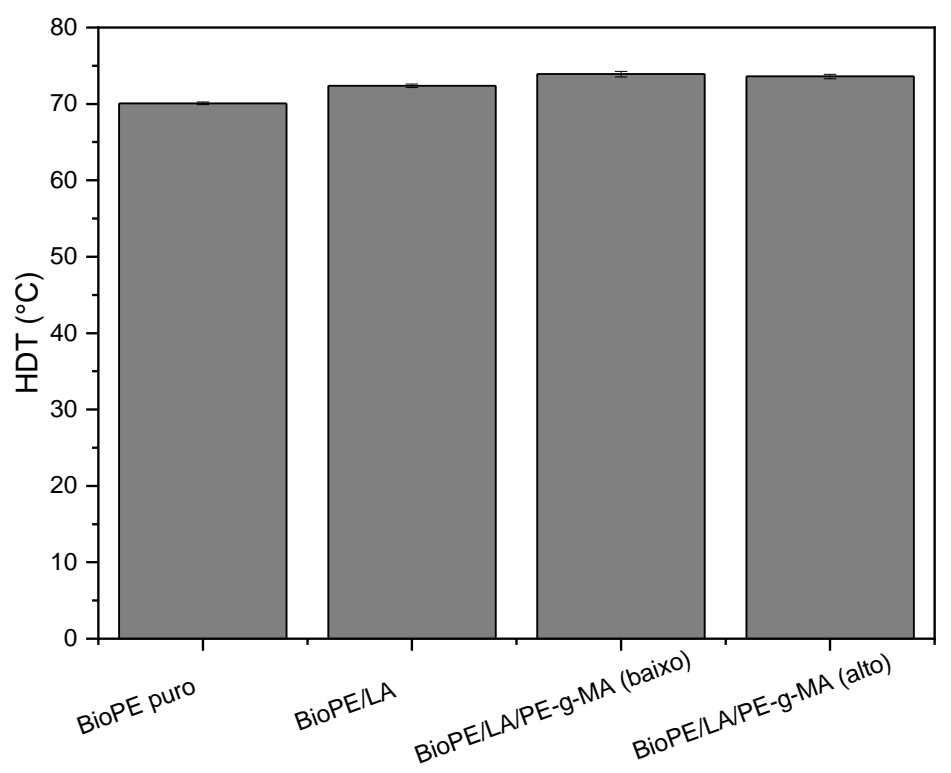

Fonte: Dados da pesquisa. 
Na Figura 8, observa-se que o valor da HDT para o BioPE foi de $70^{\circ} \mathrm{C}$, indicando uma média estabilidade termomecânica. A adição do línter (LA) aumentou de maneira sutil a resistência termomecânica do BioPE, uma vez que todos os compostos apresentaram HDT, em média, $4^{\circ} \mathrm{C}$ superior ao BioPE. A incorporação de uma carga rígida no BioPE contribuiu para o correspondente aumento dessa propriedade, corroborando os resultados do módulo elástico. Aparentemente, a influência do compatibilizante PE-g-MA e do grau de enxertia não foi significativa nessa propriedade de HDT, uma vez que os compostos estão dentro da margem de erro experimental.

Takemori (1979) indicou que a temperatura de deflexão térmica é influenciada pela rigidez e pelo grau de cristalinidade, com tendência de aumentar com o acréscimo dessas propriedades. Ao que tudo indica, o aumento na HDT dos compostos BioPE/LA e BioPE/LA/PE-g-MA está relacionado, também, com o aumento do grau de cristalinidade, como verificado em DSC.

\section{Temperatura de Amolecimento Vicat (TAV)}

A temperatura de amolecimento Vicat tem relevância em termos técnicos, servindo como referência em controle de qualidade de materiais poliméricos (Luna et al., 2020). A Figura 9 apresenta os resultados de TAV do BioPE, do BioPE/LA e dos compostos BioPE/LA/PE-g-MA. Verifica-se um acréscimo discreto na temperatura de amolecimento Vicat de todos os compostos, em comparação ao BioPE. Presume-se que, ao adicionar uma carga rígida, a matriz de BioPE tenha favorecido um aumento na dureza, provocando ganhos sutis na resistência termomecânica, corroborando o grau de cristalinidade obtido por DSC. Comparando os compostos, a temperatura de amolecimento Vicat não sofreu alterações significativas e, nesse caso, dentro da margem de erro experimental, obtiveram-se propriedades comparáveis entre si.

Figura 9. Temperatura de amolecimento Vicat (TAV) do BioPE e dos compostos.

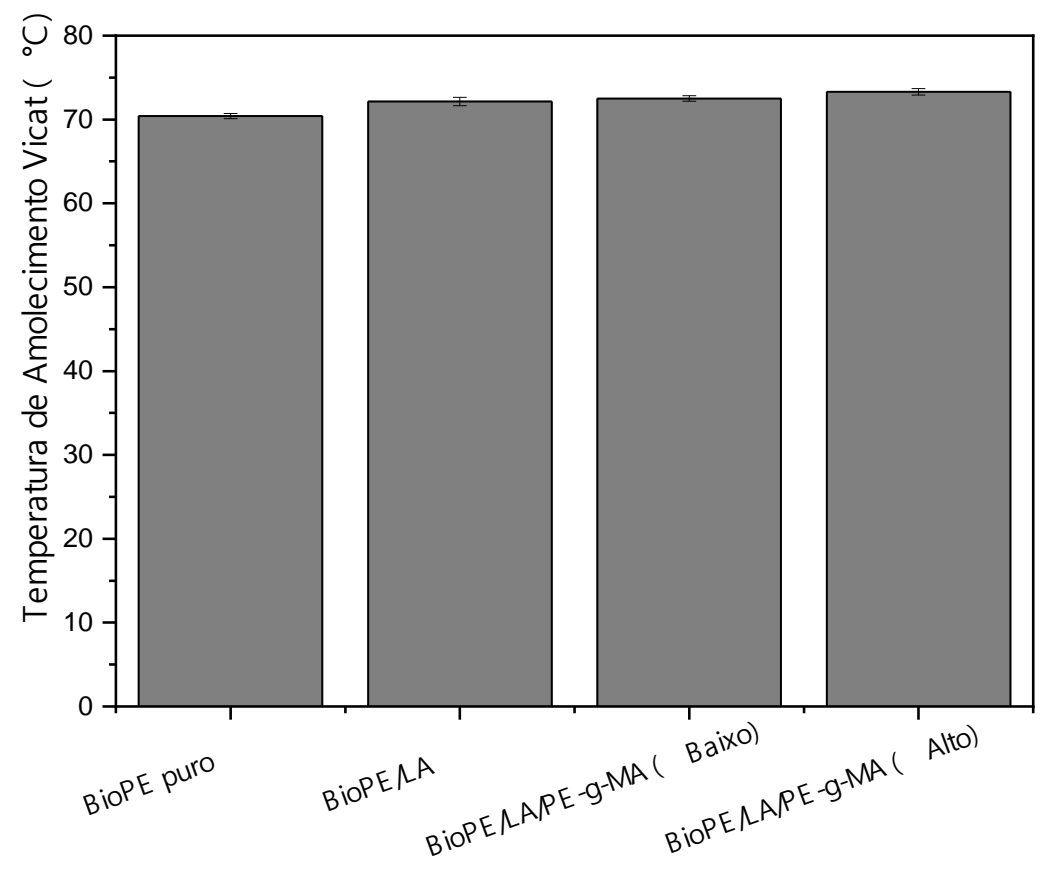

Fonte: Dados da pesquisa. 


\section{Conclusões}

Compostos de biopolietileno/línter de algodão compatibilizados com PE-g-MA foram preparados e caracterizados. Observou-se que um maior grau de enxertia de anidrido maleico no compatibilizante aprimorou as propriedades, em decorrência de aumentar a estabilidade morfológica, com partículas conectadas à matriz de BioPE e uma redução significativa das partículas arrancadas. A maior interação dos compostos BioPE/línter/PE-g-MA favoreceu à nucleação de cristais e, como consequência, aumentou o grau de cristalinidade, impactando diretamente em um ganho sutil na resistência termomecânica. Os resultados indicam, também, uma boa perspectiva para o desenvolvimento desses compostos com maiores teores de línter de algodão, valorizando um subproduto da cultura algodoeira. Ao mesmo tempo, esses compostos contribuem para a geração de novos materiais com boas propriedades e com um ciclo ambiental mais amigável.

\section{Agradecimentos}

Os autores agradecem à UFCG pela infraestrutura dos laboratórios, à Embrapa Agroindústria Tropical/Fortaleza/CE, à Polybond, à CAPES/PNPD, ao Conselho Nacional de Desenvolvimento Científico e Tecnológico - CNPq e à Coordenação de Aperfeiçoamento de Pessoal de Nível Superior - Brasil (CAPES) - Código de Financiamento 001.

\section{Referências}

Arabeche, K., Abdelmalek, F., Delbreilh, L., Zair, L., \& Berrayah, A. (2020). Physical and rheological properties of biodegradable poly(butylene succinate)/alfa fiber composites. Journal of Thermoplastic Composite Materials. https://doi.org/10.1177/0892705720904098.

Baptista, C. A., \& Canevarolo, S. V. (2019). Grafting polypropylene over hollow glass microspheres by reactive extrusion. Polímeros, 29(3), 1-9.

Beydoun, K., \& Klankermayer, J. (2020). Efficient plastic waste recycling to value-added products by integrated biomass processing. ChemSusChem, 13(3), $488-492$.

Bezerra, E. B., França, D. C., Morais, D. D. S., Siqueira, D. D., Araújo, E. M., \& Wellen, R. M. R. (2019a). Toughening of bio-PE upon addition of PCL and PEgAA. REM - International Engineering Journal, 72(3), 469-478.

Bezerra, E. B., França, D. C., Morais, D. D. S., Silva, I. D. S., Siqueira, D. D., Araújo, E. M., \& Wellen, R. M. R. (2019b). Compatibility and characterization of Bio-PE/PCL blends. Polímeros, 29(2), 1-15.

Bezerra, E. B., França, D. C., Morais, D. D. S., Araújo, E. M., Rosa, M. F., \& Oliveira, A. D. (2015). Estudo das propriedades mecânicas e termomecânicas de compósitos de poli(c-caprolactona)/nanolínter. Revista Eletrônica de Materiais e Processos, 10(1), 16-20.

Brito, G. F., Agrawal, P., Araújo, E. M., \& Mélo, T. J. A. (2012). Polylactide/Biopolyethylene bioblends. Polímeros, 22(5), $427-429$.

Bosenbecker, M. W., Croche, S., Cholant, G., Rosa, P., Passador, F., Marini, J., \& Oliveira, A. (2018). Propriedades térmicas de compósitos de polietileno de alta densidade reforçados com celulose. Revista Brasileira de Engenharia e Sustentabilidade, 5(especial), 7-12.

Castro, D. O., Passador, F., Filho, A. R., \& Frollini, E. (2017). Use of castor and canola oils in "biopolyethylene" curauá fiber composites. Composites Part A: Applied Science and Manufacturing, 95(4), 22-30.

Castro, D. O., Filho, A. R., \& Frollini, E. (2012). Materials prepared from biopolyethylene and curaua fibers: Composites from biomass. Polymer Testing, $31(7), 880-888$

Castro, D. O., Frollini, E., Marini, J., \& Filho, A. R. (2013). Preparação e caracterização de biocompósitos baseados em fibra de curauá, biopolietileno de alta densidade (BPEAD) e polibutadieno líquido hidroxilado (PBHL). Polímeros, 23(1), 65-73.

Cataldi, P., Cassinelli, M., Guerrero, J. A. H., Puyol, S. G., Naderizadeh, S., Athanassiou, A., \& Caironi, M. (2020). Green biocomposites for thermoelectric wearable applications. Advanced Functional Materials, 30(3), 1-10.

Catto, A. L., Stefani, B. V., Ribeiro, V. F., \& Santana, R. M. C. (2014). Influence of coupling agent in compatibility of post-consumer HDPE in thermoplastic composites reinforced with eucalyptus fiber. Materials Research, 17(1), 1-7.

Cicconi, P. (2020). Eco-design and eco-materials: An interactive and collaborative approach. Sustainable Materials and Technologies, $23(4), 1-24$.

Daghigh, V., Júnior, T. E. L., Daghigh, H., Gu, G., Baghaei, K. T., Horstemeyer, M. F., Júnior, C. U. P. (2020). Heat deflection temperatures of bio-nanocomposites using experiments and machine learning predictions. Materials Today Communications, 22(3), 100789.

Dong, C. (2017). Review of natural fibre-reinforced hybrid composites. Journal of Reinforced Plastics and Composites, 37(5), 331-348. 
Ferreira, E. S. B., Luna, C. B. B., Araújo, E. M., Siqueira, D. D., \& Wellen, R. M. R. (2019). Polypropylene/wood powder composites: Evaluation of PP viscosity in thermal, mechanical, thermomechanical, and morphological characters. Journal of Thermoplastic Composite Materials. https://doi.org/10.1177/0892705719880958.

Ferreira, E. S. B., Luna, C. B. B., Siqueira, D. D., Araújo, E. M., França, D. C., \& Wellen, R. M. R. (2021). Annealing Effect on PLA/EVA Blends Performance. Journal of Polymers and the Environment. https://doi.org/10.1007/s10924-021-02220-4.

Firmino, H. C. T., Chagas, T. F., Melo, P. M. A., \& Silva, L. B. (2017). Caracterização de compósitos particulados de polietileno de alta densidade/pó de concha de molusco. Matéria (Rio J.), 22(4), 1-12.

Gandara, M., Gonçalves, A. R., \& Saron, C. (2017). Compósitos de PET reciclado com fibra de cana-de-açúcar tratada por explosão a vapor. Matéria (Rio J.), $22(4), 1-10$

Hoffmannab, R., Morais, D. D. S., Braz, C. J. F., Haaga, Wellen, R. M. R., Canedo, E. L., Carvalho, L. H., \& Koschek, K. (2019). Impact of the natural filler babassu on the processing and properties of PBAT/PHB films. Composites Part A: Applied Science and Manufacturing, 124(9), 1-9.

Jesus, L. C. C., Luz, S. M., Leão, R. M., Zattera, A. J., \& Amico, S. C. (2019). Comportamento térmico de compósitos de poliestireno reciclado reforçado com celulose de bagaço de cana. Matéria (Rio J.), 24 (3), 1-11.

Júnior Padilha, E. J., \& Zard, C. L. (2010). Comportamento mecânico e reológico de compósitos de polipropileno e fibras de bananeira: Influência do teor de fibra. Revista Eletrônica de Materiais e Processos, 5(1), 10-16.

Kim, J., \& Cho, D. (2020). Effects of Waste Expanded Polypropylene as Recycled Matrix on the Flexural, Impact, and Heat Deflection Temperature Properties of Kenaf Fiber/Polypropylene Composites. Polymers, 12(11), 2578, 2020.

Kotik, H. G. (2019). Fibras naturais e compósitos reforçados com fibras naturais: a motivação para sua pesquisa e desenvolvimento. Matéria (Rio J.), 24 (3), $1-3$.

Marinho, V. A. D., Almeida, T. G., Carvalho, L. H., \& Canedo, E. L. (2018). Aditivação e biodegradação de compósitos PHB/babaçu. Revista Eletrônica de Materiais e Processos, 13(1), 37-41.

Mei, L. H., \& Oliveira, N. (2017). Caracterização de um compósito polimérico biodegradável utilizando poli (E-caprolactona) e borra de café. Polímeros, 27(número especial), 99-109.

Montanes, N., Sanoguera, D. G., Segui, V. J., Fenollar, O., \& Boronat, T. (2018). Processing and characterization of environmentally friendly composites from biobased polyethylene and natural fillers from thyme herbs. Journal of Polymers and the Environment, 26(5), 1218-1230.

Nery, T. B. R., Santos, Z. I. G., \& José, N. M. (2018). Desenvolvimento e caracterização de biocompósitos de polihidroxibutirato e fibra de bananeira. Matéria (Rio J.), 23(4), 1-9.

Poletto, M. (2017a). Compósitos termoplásticos com madeira - uma breve revisão. RICA, 2(4), 42-48.

Poletto, M. (2017b). Mechanical, dynamic mechanical and morphological properties of composites based on recycled polystyrene filled with wood flour wastes. Maderas. Ciencia y tecnologia, 19(4), 433-442.

Poletto, M. (2018). Maleated soybean oil as coupling agent in recycled polypropylene/wood flour composites: Mechanical, thermal, and morphological properties. Journal of Thermoplastic Composite Materials, 32(8), 1056-1067.

Rabello, M. S. Aditivação de polímeros Artliber Editora, 2000.

Ramos, R. R. F., Siqueira, D. D., Wellen, R. M. R., Leite, I. F., Glenn, G. M., \& Medeiros, E. S. (2019). Development of green composites based on polypropylene and corncob agricultural residue. Journal of Polymers and the Environment, 27(5), 1677-1685.

Ramos, S. M. L. S., Carvalho, L. H., Spieth, E., \& Rivadula, R. S. M. (1993). Efeitos da estabilização do Polipropileno nas propriedades térmicas, mecânicas e termo-mecânicas de compósitos de polipropileno/atapulgita. Polímeros, 3(4), 26-31.

Redighieri, K. A., \& Costa, D. A. (2008). Compósitos de polietileno reciclado e partículas de madeira de reflorestamento tratadas com polietileno modificado. Polímeros, 18(1), 5-11.

Santos, E. B., Passador, F. R., \& Montagna, L. S. (2020). Influência de fatores ambientais nas propriedades mecânicas de biocompósitos de PLA reforçado com fibra de coco e borra de café. Tecno-Lógica, 24(1), 93-102.

Santos, E. F., Moresco, M., Rosa, S. M. L., \& Nachtigall, S. M. B. (2010). Extrusão de compósitos de PP com fibras curtas de coco: efeito da temperatura e agentes de acoplamento. Polímeros, 20(3), 215-220.

Santos Filho, E. A., Luna, B. B. B., Siqueira, D. D., Nogueira, J. A. S., \& Araújo, E. M. (2021). Reuse of carbon fiber waste to produce composites with polypropylene. The effect of styrene-(ethylene-butylene)-styrene grafted with maleic anhydride and ethylene-propylene-diene grafted with maleic anhydride copolymers. Polymer Composites. Epub ahead of print. https://doi.org/10.1002/pc.26295.

Seixas, J. N., Granada, J. E., Melo, C. C. N., Silva, G. E. H., Passador, F. R., Cholant, G. M., Oliveira, A. D., Beatrice, C. A. G., Gonçalves, M. R. F., \& Carreño, N. N. L. V. (2018). Compósitos de polipropileno reforçados com fibras naturais do talo da banana em diferentes granulometrias. Revista Brasileira de Engenharia e Sustentabilidade, 5(especial), 32-37.

Silva, C. C., Júnior, R. C. S. F., Ford, E. T. L. C., Dantas, C. M., Santos, J. K. D., \& Aquino, E. M. F. (2018). Mechanical behavior and water absorption in sisal/glass hybrid composites. Matéria (Rio J.), 23(4), 1-10. 
Silva, F. A., Luna, C. B. B., Siqueira, D. D., Ferreira, E. S. B., \& Araújo, E. M. (2021). From Waste to Reuse: Manufacture of Ecological Composites Based on Biopolyethylene/wood Powder with PE-g-MA and Macaíba Oil. Journal of Polymers and the Environment. https://doi.org/10.1007/s10924-021-02256-6.

Siqueira, D. D., Luna, C. B. B., Araújo, E. M., Filho, E. A. S., Morais, D. D. S., \& Wellen, R. M. R. (2019a). Biodegradable compounds of poly (Ecaprolactone)/montmorillonite clays. Materials Research, 22(suppl. 1), 1-10.

Siqueira, D. D., Luna, C. B. B., Araújo, E. M., Ferreira, E. S. B., \& Wellen, R. M. R. (2019b). Biocomposites based on PCL and macaiba fiber. Detailed characterization of main properties. Materials Research Express, 6(9), 1-12.

Siqueira, D. D., Luna, C. B. B., Ferreira, E. S. B., Araújo, E. M., \& Wellen, R. M. R. (2020). Tailored PCL/Macaíba fiber to reach sustainable biocomposites. Journal of Materials Research and Technology, 9(5), 9691-9708.

Spadetti, C., Filho, E. A. S., Sena, G. L., \& Melo, C. V. P. (2017). Propriedades térmicas e mecânicas dos compósitos de Polipropileno pós-consumo reforçados com fibras de celulose. Polímeros, 27(especial), 84-90.

Liu, T., Lei, Y., Wang, Q., Lee, S., Wu, Q. (2013). Effect of Fiber Type and Coupling Treatment on Properties of High-Density Polyethylene/Natural Fiber Composites. BioResources, 8(3), 4619-4632.

Luna, C. B. B., Ferreira, E. S. B., Silva, L. J. M. D., Silva, W. A., Araújo, E. M., \& Melo, J. B. C. S. (2019a). Blends with technological potential of copolymer polypropylene with polypropylene from post-consumer industrial containers. Materials Research Express, 6(12), 1-13.

Luna, C. B. B., Siqueira, D. D., Araújo, E. M., \& Wellen, R. M. R. (2019b). Tailoring PS/PPrecycled blends compatibilized with SEBS. Evaluation of rheological, mechanical, thermomechanical and morphological characters. Materials Research Express, 6(7), 1-16.

Luna, C. B. B., Siqueira, D. D., Ferrera, E. S. B., Araújo, E. M., \& Wellen, R. M. R. (2019c). Reactive compatilization of PCL/WP upon addition of PCL-MA. Smart option for recycling industry. Materials Research Express, 6(12), 1-15.

Luna, C. B. B., Ferreira, E. S. B., Siqueira, D. D., Silva, W. A., Araújo, E. M., \& Wellen, R. M. R. (2019d). Tailoring performance of PP/HIPS/SEBS through blending design. Materials Research Express, 6(11), 1-17.

Luna, C. B. B., Araújo, E. M., Siqueira, D. D., Morais, D. D. S., Filho, E. A. S., \& Fook, M. V. L. (2020). Incorporation of a recycled rubber compound from the shoe industry in polystyrene: Effect of SBS compatibilizer contente. Journal of Elastomers \& Plastics, 52(1) 3-28.

Luna, C. B. B., Ferreira, E. S. B., Nogueira, J. A. S., Araújo, E. M., Nascimento, E. P., \& Melo, J. B. C. A. (2021). Biopolyethylene/Morinda citrifolia cellulosic biocomposites: The impact of chemical crosslinking and PE-g-MA compatibilizer. Polymer Composites. Epub ahead of print. https://doi.org/10.1002/pc.26320.

Morais, D. D. S., Siqueira, D. D., Luna, C. B. B., Araújo, E. M., Bezerra, E. B., Wellen, R. M. R. (2019). Grafting maleic anhydride onto polycaprolactone: influence of processing. Materials Research Express, 6(5), 055315.

Taghavi, S. K., Shahrajabian, H., \& Hosseini, H. M. (2018). Detailed comparison of compatibilizers MAPE and SEBS-g-MA on the mechanical/thermal properties, and morphology in ternary blend of recycled PET/HDPE/MAPE and recycled PET/HDPE/SEBS-g-MA. Journal of Elastomers \& Plastics, 50(1), $13-35$.

Takemori, M.T. (1979). Towards an understanding of the heat distortion temperature of thermoplastics. Polym Eng Sci, 19(15), 1104-1109.

Wis, A. A., Kodal, M., Ozturk, S., \& Ozkoc, G. (2020). Overmolded polylactide/jute-mat eco-composites: A new method to enhance the properties of natural fiber biodegradable composites. Journal of Applied Polymer Science, 137(20), 1-10.

Yang, H. S., Wolcott, M. P., Kim, H. S., Kim, S., \& Kim, H. J. (2006). Properties of lignocellulosic material filled polypropylene bio-composites made with different manufacturing processes. Polymer Testing, 25(5), 668-676.

Zhang, Q., Lu, W., Zhou, L., Zhang, D., Cai, H., \& Lin, X. (2019). Tensile and flammability characterizations of corn straw slagging/high-density polyethylene composites. Journal of Thermoplastic Composite Materials. https://doi.org/10.1177/0892705719830459. 